\title{
A Two-Period Newsvendor Setting with Product Extension and Shifting Demand Concerns
}

\author{
Kuo-Hsien Wang ${ }^{1}$, Che-Tsung Tung ${ }^{2, *}$, Yuan-Chih Huang ${ }^{3}$ \\ ${ }^{1}$ Dept. of Business Administration, Takming University of Science and Technology, Taiwan \\ ${ }^{2}$ Dept. of International Trade, Takming University of Science and Technology, Taiwan \\ ${ }^{3}$ Dept. of Business Administration, Takming University of Science and Technology, Taiwan \\ *Corresponding author:dennistung@takming.edu.tw
}

Copyright $@ 2013$ Horizon Research Publishing All rights reserved.

\begin{abstract}
The objective of this study is to optimize an overall expected profit in a two-period newsvendor setting by jointly determining these two items' order sizes and selling prices. The item in the second period is a product extension of the item in the first period. An inadequate inventory is intentionally placed in the first period in order to stimulate more sales in the second period. The first item's unsatisfied demand is assumed to be transferred to the second selling period with a certain shifting demand rate. The two items' stochastic demand is assumed to be in a linear-additive form consisting of a deterministic demand and an error demand that is exponentially distributed. Including the classical newsvendor model as a special case, we first propose a pertinent model that overshadows the classical newsvendor setting in terms of profit performances, and surprisingly find that the first item's error demand should be completely sacrificed when facing a high shifting demand rate. Afterwards, we modify the model to identify that our inadequate stock strategy is indeed practicable, and then extend the model to predetermine the two items' selling prices that ensure the existence of maximum of the expected profit. Possible triggered effects, such as an increasing primary demand, an increasing error demand and a cheaper purchasing cost in the second period, are also illustrated by examples.
\end{abstract}

Keywords Newsvendor, Product extension, Shifting demand, Newsboy, EOQ

\section{Introduction}

A prevalent phenomenon in recent markets is to connect newsvendor-type items with the concept of product extension and the strategy of inadequate stock. That is, a series of homogeneous items are introduced one after another, during where a shortage of a prior item is intentionally made in a purpose of stimulating more unexpected buyers for its next item; items such as a series of iPhone, iPad and Galaxy are among the illustrations; both companies of which occupy a very large smartphone and tablet computer market shares. Therefore, instead of the classical single-period newsvendor setting, a multi-period for serial homogeneous items incorporating with a scheme of shortage-making has been becoming a very important market approach nowadays.

Referring to Arcelus et al. [1], a stochastic demand consisting of a deterministic demand and an error demand is a characteristic of any specific newsvendor item, in which linear-additive $\quad \alpha-\beta p+\varepsilon \quad, \quad$ linear-multiplicative $(\alpha-\beta p) \varepsilon \quad, \quad$ iso-elastic-additive $\quad \alpha p^{-\beta}+\varepsilon \quad$ and iso-elastic-multiplicative $\left(\alpha p^{-\beta}\right) \varepsilon$ are four characteristics broadly accepted in the existing literature. Regarding the linear-additive form $\alpha-\beta p+\varepsilon, \alpha-\beta p$ is the deterministic demand with that $\alpha$ is a primary demand, $\beta$ is a price sensitivity and $p$ is a selling price, and $\varepsilon$ is an error demand usually associated with an uniform distribution [2], a normal distribution [3], an exponential distribution [4] or a Poisson distribution [5].

Generally, the primary demand $\alpha$ could be estimated according to information obtained by means of observation, surveys or experimentation in the markets. And its size largely depends on company goodwill, product quality, product applicability and the degree of customers' acceptance. This therefore infers that the primary demand could be improved according to its previous and homogeneous item's sales status and market responses, which consequently accounts for why combing product extension with a multi-period setting plays such an important role in modern marketing strategy. A "product extension" is a new version of an item with different dimensions or additional or upgraded features in comparison with its previous introduced item.

Halati and $\mathrm{He}$ [6] studied a single-period newsvendor problem with quantity based fixed incentives in a decentralized supply chain setting, of which their results was then modified to a multi-period setting. But they did not take the shortage-making strategy into account at that time. In contrast, a number of extant publications have been 
focusing on a single period setting, such as Lau and Lau [7] investigated a manufacturer's pricing strategy and return policy in a framework of a single-period commodity. Chung and Flynn [8] tackled a newsboy problem considering reactive production in a single selling period. Das and Maiti[9] handled a bi-level newsboy problem in two substitutable items under a condition of capital cost. Bose and Anand[10] addressed themselves to a newsvendor problem on returns policies with exogenous price. Serin[11] developed competitive newsvendor problems with the same Nash and Stackelberg solutions. Shi and Chen [12]analyzed Pareto-optimal contracts for a newsvendor supply chain with satisficing objectives, and Yang et al. [13] explored optimal ordering and pricing decisions for a newsvendor problem with revenue-target-oriented and profit-target-oriented objectives. Nonetheless, not all single period inventory settings are categorized as the classical newsvendor problem. Wang and Tung [14], motivated by a behavior of population growth rate, constructed a time-dependent demand model for gradually obsolescent products that face a short lifespan. You and Chen [15] dealt with the related problem, jointly determining order size and price for perishable products over a finite time planning horizon with an assumption of a time and price dependent demand. Both of the papers mentioned above belong to single period EOQ, but not the classical newsvendor problem.

In this study we attempt to connect a two-period newsvendor setting with product extension and inadequate stock strategy. The item in the second period is a product extension of the item in the first period. An inadequate order size is purposely placed in the first period so as that some of the first item's unsatisfied demand will transfer to the second period in a purpose of enticing much more sales toward the second item. Three merits of our system comprise that (1) the first item's sales information could provide a revision guideline to ameliorate the second item's primary demand; (2) for those shifting demand, a higher profit margin in the second period is usually allowed, and this will help improve overall profit performances; (3) possible effects, such as an increasing primary demand, an increasing error demand and even a cheaper item 2's purchasing cost due to manufacturer's promotional techniques, manufacturing machine's improvement and staff technology promotion, would likely be simultaneously triggered in the second period to stimulate more buyers and create more profit as well. Also, the following questions will be respectively resolved in the course of this study. (1) How to determine the two order sizes and the two selling prices that maximize the overall expected profit? (2) Whether or not our system is always outperforming the classical newsvendor setting from profit performance's standpoint? (3) How would the first item's order size influence the profit performances? (4) Is there any restriction of the two selling prices to ensure that our profit is always better off than that in the classical newsvendor setting? (5) What will the three triggered effects impact on the profit?

The remainder of this study is organized as follows. Assumptions and notation are given in Section 2 where a relevant model responding to our main concerns is proposed. Its theoretical analysis to optimize the overall profit is conducted in Section 3, along with two sub-sections that respectively illustrate the correlation between the profit and the first item's order size, as well as the impacts of the two selling prices on the profit performances. Numerical examples are conducted in Section 4, followed by many managerial insights and analyses of impact on the profit. Finally, a summary and contributions of the study and potential directions for further researches are presented in Section 5 to complete the study.

\section{The Model}

The scenario of this study is described as follows. Two homogeneous newsvendor-type items, say item 1 and item 2 , will respectively be sold in two distinct selling periods. Item 2 is a product extension of item 1 . An inadequate order size of item 1 is intentionally placed in the first period so as that its unsatisfied demand will shift to the second selling period with a known shifting demand rate $r, 0 \leq r \leq 1$. For simplicity, we let $h$ is the unit overage cost throughout the entire system. Also, based on Yang et al. [13], zero goodwill cost is assumed, and the underage cost is just the loss of revenue. For item $i, i=1,2, D_{i}=\alpha_{i}-\beta_{i} p_{i}$ is the deterministic demand with primary demand $\alpha_{i}$, consumer's price-sensitivity $\beta_{i}$ and price $p_{i} ; \varepsilon_{i} \in[0, \infty)$ is an error demand exponentially distributed with pdf $f_{i}(\cdot), \operatorname{cdf} F_{i}(\cdot)$ and mean $\mu_{i}$. The item $i$ 's stochastic demand is then assumed to be in a linear-additive form of $x_{i}=D_{i}+\varepsilon_{i}$, whose unit purchasing $\operatorname{cost}$ is $c_{i}$.

For the first period, according to Thowsen[16] and Petruzzi and Dada [17], we assume item 1's order size by $Q_{1}=D_{1}+z_{1}$ where $z_{1}$ is responding to the error demand $\varepsilon_{1}$; thus its corresponding profit is then calculated by

$$
\begin{aligned}
& \pi_{1}=\left(p_{1}-c_{1}\right)\left(D_{1}+\varepsilon_{1}\right)-\left(c_{1}+h\right)\left(z_{1}-\varepsilon_{1}\right)^{+}- \\
& \left(p_{1}-c_{1}\right)\left(\varepsilon_{1}-z_{1}\right)^{+}
\end{aligned}
$$

where $(\cdot)^{+}=\max \{\cdot, 0\}$. And its expected profit is given by

$$
\begin{aligned}
& E_{1}=\left(p_{1}-c_{1}\right)\left(D_{1}+\mu_{1}\right)-\left(c_{1}+h\right) \Lambda_{1}\left(z_{1}\right)- \\
& \left(p_{1}-c_{1}\right) \Theta_{1}\left(z_{1}\right)
\end{aligned}
$$

where

$$
\Lambda_{1}\left(z_{1}\right)=\int_{0}^{z_{1}}\left(z_{1}-\varepsilon_{1}\right) f_{1}\left(\varepsilon_{1}\right) d \varepsilon_{1}
$$

and 


$$
\Theta_{1}\left(z_{1}\right)=\int_{z_{1}}^{\infty}\left(\varepsilon_{1}-z_{1}\right) f_{1}\left(\varepsilon_{1}\right) d \varepsilon_{1}
$$

As for the second period, Eq.(2) reveals that $\Theta_{1}\left(z_{1}\right)$ is the expected amount of unsatisfied demand in the first period; thus according to the assumption, $r \Theta_{1}\left(z_{1}\right)$ is the amount of that unsatisfied demand that will shift to the second period, leading the item 2 to a total demand of $x_{2}+r \Theta_{1}\left(z_{1}\right)=D_{2}+r \Theta_{1}\left(z_{1}\right)+\varepsilon_{2}$. Likewise, applying the aforementioned scheme, let $Q_{2}=D_{2}+r \Theta_{1}\left(z_{1}\right)+z_{2}$ be item 2's order size whose profit is then computed by

$$
\begin{aligned}
& \pi_{2}=\left(p_{2}-c_{2}\right)\left(D_{2}+r \Theta_{1}\left(z_{1}\right)+\varepsilon_{2}\right)- \\
& \left(c_{2}+h\right)\left(z_{2}-\varepsilon_{2}\right)^{+}-\left(p_{2}-c_{2}\right)\left(\varepsilon_{2}-z_{2}\right)^{+}
\end{aligned}
$$

And its expected profit is given by

$$
\begin{aligned}
& E_{2}=\left(p_{2}-c_{2}\right)\left(D_{2}+r \Theta_{1}\left(z_{1}\right)+\mu_{2}\right)- \\
& \left(c_{2}+h\right) \Lambda_{2}\left(z_{2}\right)-\left(p_{2}-c_{2}\right) \Theta_{2}\left(z_{2}\right)
\end{aligned}
$$

Where

$$
\Lambda_{2}\left(z_{2}\right)=\int_{0}^{z_{2}}\left(z_{2}-\varepsilon_{2}\right) f_{2}\left(\varepsilon_{2}\right) d \varepsilon_{2}
$$

and

$$
\Theta_{2}\left(z_{2}\right)=\int_{z_{2}}^{\infty}\left(\varepsilon_{2}-z_{2}\right) f_{2}\left(\varepsilon_{2}\right) d \varepsilon_{2}
$$

Adding the two expected profits above together generates an overall expected profit for our two-period setting as follows.

$$
\begin{gathered}
E\left(z_{1}, z_{2}, p_{1}, p_{2}\right)=\sum_{i=1}^{2}\left(p_{i}-c_{i}\right)\left(D_{i}+\mu_{i}\right)- \\
\sum_{i=1}^{2}\left(c_{i}+h\right) \Lambda_{i}\left(z_{i}\right)-\sum_{i=1}^{2}\left(p_{i}-c_{i}\right) \Theta_{i}\left(z_{i}\right) \\
+\left(p_{2}-c_{2}\right) r \Theta_{1}\left(z_{1}\right)
\end{gathered}
$$

Obviously, the first term of Eq.(5) stands for the two items' expected profits; the second term represents an expected overage cost; the third term implies an expected loss resulted from unsatisfied demand and the last term is an extra expected profit in the second period because of the shifting demand $r \Theta_{1}\left(z_{1}\right)$. We note that the derived $E\left(z_{1}, z_{2}, p_{1}, p_{2}\right)$ could apply to the classical two-period newsvendor setting if $r=0$.

\section{Analysis}

Recall that $E\left(z_{1}, z_{2}, p_{1}, p_{2}\right)$ is a function of $z_{1}, z_{2}, p_{1}$ and $p_{2}$. For convenience, it will be replaced with $E$ hereafter. So, respectively taking its first- and second-order partial derivatives yields the following results.

$$
\begin{aligned}
& \frac{\partial E}{\partial z_{1}}=\left(p_{1}-c_{1}\right)-r\left(p_{2}-c_{2}\right)+ \\
& {\left[r\left(p_{2}-c_{2}\right)-\left(p_{1}+h\right)\right] F_{1}\left(z_{1}\right)} \\
& \frac{\partial E}{\partial z_{2}}=\left(p_{2}-c_{2}\right)-\left(p_{2}+h\right) F_{2}\left(z_{2}\right) \\
& \frac{\partial E}{\partial p_{1}}=-2 \beta_{1} p_{1}+\alpha_{1}+\mu_{1}+c_{1} \beta_{1}-\Theta_{1}\left(z_{1}\right) \\
& \frac{\partial E}{\partial p_{2}}=-2 \beta_{2} p_{2}+\alpha_{2}+\mu_{2}+c_{2} \beta_{2}-\Theta_{2}\left(z_{2}\right)+r \Theta_{1}\left(z_{1}\right)
\end{aligned}
$$

Also,

$$
\begin{gathered}
\frac{\partial^{2} E}{\partial z_{1}^{2}}=\left[r\left(p_{2}-c_{2}\right)-\left(p_{1}+h\right)\right] f_{1}\left(z_{1}\right) \\
\frac{\partial^{2} E}{\partial z_{2}^{2}}=-\left(p_{2}+h\right) f_{2}\left(z_{2}\right)
\end{gathered}
$$

$$
\begin{gathered}
\frac{\partial^{2} E}{\partial p_{1}^{2}}=-2 \beta_{1}, \frac{\partial^{2} E}{\partial p_{2}^{2}}=-2 \beta_{2}, \frac{\partial^{2} E}{\partial z_{1} \partial z_{2}}=0, \\
\frac{\partial^{2} E}{\partial z_{1} \partial p_{1}}=1-F_{1}\left(z_{1}\right), \frac{\partial^{2} E}{\partial z_{1} \partial p_{2}}=r\left(F_{1}\left(z_{1}\right)-1\right), \\
\frac{\partial^{2} E}{\partial z_{2} \partial p_{1}}=0, \frac{\partial^{2} E}{\partial z_{2} \partial p_{2}}=1-F_{2}\left(z_{2}\right)
\end{gathered}
$$

and $\frac{\partial^{2} E}{\partial p_{1} \partial p_{2}}=0$. Its corresponding Hessian matrix is then given by

$$
H=\left[\begin{array}{cccc}
\left(r\left(p_{2}-c_{2}\right)-\left(p_{1}+h\right)\right) f_{1}\left(z_{1}\right) & 0 & 1-F_{1}\left(z_{1}\right) & r\left(F_{1}\left(z_{1}\right)-1\right) \\
0 & -\left(p_{2}+h\right) f_{2}\left(z_{2}\right) & 0 & 1-F_{2}\left(z_{2}\right) \\
1-F_{1}\left(z_{1}\right) & 0 & -2 \beta_{1} & 0 \\
r\left(F_{1}\left(z_{1}\right)-1\right) & 1-F_{2}\left(z_{2}\right) & 0 & -2 \beta_{2}
\end{array}\right]
$$

And its principal minors are

$$
\begin{aligned}
& \left|H_{11}\right|=-\left(\left(p_{1}+h\right)-r\left(p_{2}-c_{2}\right)\right) f_{1}\left(z_{1}\right) \\
& \left|H_{22}\right|=\left(\left(p_{1}+h\right)-r\left(p_{2}-c_{2}\right)\right) f_{1}\left(z_{1}\right)\left(p_{2}+h\right) f_{2}\left(z_{2}\right) \\
& \left|H_{33}\right|=-\left[2 \beta_{1}\left(\left(p_{1}+h\right)-r\left(p_{2}-c_{2}\right)\right) f_{1}\left(z_{1}\right)-\left(1-F\left(z_{1}\right)\right)^{2}\right] \\
& \left(p_{2}+h\right) f_{2}\left(z_{2}\right)
\end{aligned}
$$

and

$$
\begin{gathered}
\left|H_{44}\right|=4 \beta_{1} \beta_{2}\left(\left(p_{1}+h\right)-r\left(p_{2}-c_{2}\right)\right) f_{1}\left(z_{1}\right)\left(p_{2}+h\right) f_{2}\left(z_{2}\right) \\
-2 \beta_{1}\left(\left(p_{1}+h\right)-r\left(p_{2}-c_{2}\right)\right) f_{1}\left(z_{1}\right)\left(1-F_{1}\left(z_{1}\right)\right)^{2}
\end{gathered}
$$




$$
\begin{gathered}
+\left(1-F_{1}\left(z_{1}\right)\right)^{2}\left(1-F_{2}\left(z_{2}\right)\right)^{2}- \\
2 \beta_{2}\left(p_{2}+h\right) f_{2}\left(z_{2}\right)\left(1-F_{1}\left(z_{1}\right)\right)^{2} \\
-2 \beta_{1} r^{2}\left(p_{2}+h\right) f_{2}\left(z_{2}\right)\left(1-F_{1}\left(z_{1}\right)\right)^{2} \\
\geq\left[2 \beta_{1}\left(\left(p_{1}+h\right)-r\left(p_{2}-c_{2}\right)\right) f_{1}\left(z_{1}\right)-\left(1-F_{1}\left(z_{1}\right)\right)^{2}\right] \\
{\left[2 \beta_{2}\left(p_{2}+h\right) f_{2}\left(z_{2}\right)-\left(1-F_{2}\left(z_{2}\right)\right)^{2}\right]} \\
-2 \beta_{1}\left(p_{2}+h\right) f_{2}\left(z_{2}\right)\left(1-F_{1}\left(z_{1}\right)\right)^{2}
\end{gathered}
$$

Clearly, the conditions of

$\left(\left(p_{1}+h\right)-r\left(p_{2}-c_{2}\right)\right) f_{1}\left(z_{1}\right)>1+\frac{1}{2 \beta_{1}}$ and

$\left(p_{2}+h\right) f_{2}\left(z_{2}\right)>\frac{1}{2 \beta_{2}-1}$ guarantee the

negative-definite $H$ since $\left|H_{11}\right|<0,\left|H_{22}\right|>0,\left|H_{33}\right|<0$ and $\left|H_{44}\right|>0$, and this proves that the $E$ is jointly

concave in $z_{1}, z_{2}, p_{1}$ and $p_{2}$.

Furthermore, from Eqs.(6)-(9), the optimal necessary condition satisfies the following equations.

$$
\begin{gathered}
F_{1}\left(z_{1}\right)=\frac{\left(p_{1}-c_{1}\right)-r\left(p_{2}-c_{2}\right)}{\left(p_{1}+h\right)-r\left(p_{2}-c_{2}\right)} \\
F_{2}\left(z_{2}\right)=\frac{p_{2}-c_{2}}{p_{2}+h} \\
p_{1}=\frac{1}{2 \beta_{1}}\left(\alpha_{1}+\mu_{1}+c_{1} \beta_{1}-\Theta_{1}\left(z_{1}\right)\right) \\
p_{2}=\frac{1}{2 \beta_{2}}\left(\alpha_{2}+\mu_{2}+c_{2} \beta_{2}-\Theta_{2}\left(z_{2}\right)+r \Theta_{1}\left(z_{1}\right)\right)
\end{gathered}
$$

In addition, the Eqs.(10)-(13) induce the following two facts regarding the optimal $z_{1}, z_{2}, p_{1}, p_{2}$. (1) $0 \leq z_{1}, z_{2}<\infty$,

$$
\frac{1}{2 \beta_{1}}\left(\alpha_{1}+c_{1} \beta_{1}\right) \leq p_{1}<\frac{1}{2 \beta_{1}}\left(\alpha_{1}+\mu_{1}+c_{1} \beta_{1}\right)
$$

and

$$
\begin{aligned}
& \frac{1}{2 \beta_{2}}\left(\alpha_{2}+c_{2} \beta_{2}\right)<p_{2}<\frac{1}{2 \beta_{2}}\left(\alpha_{2}+\mu_{2}+c_{2} \beta_{2}+r \mu_{1}\right) \\
& \text { (2) } \frac{d p_{1}}{d z_{1}}>0, \frac{\partial p_{2}}{\partial z_{2}}>0, \frac{\partial p_{2}}{\partial z_{1}}<0 \text {, indicating that } p_{1}
\end{aligned}
$$

positively correlates with $z_{1}, p_{2}$ positively correlates with $z_{2}$ but negatively correlates with $z_{1}$.

Utilizing the two facts obtained, we now can prove the unique existence of the optimal values $z_{1}, z_{2}, p_{1}, p_{2}$ in Eqs.(10)-(13) as follows. First, we set $p_{1}^{0}=\frac{1}{2 \beta_{1}}\left(\alpha_{1}+c_{1} \beta_{1}\right)$, a possible minimum $p_{1}$ associated with $z_{1}=0$, and then set $p_{2}^{0}=\frac{1}{2 \beta_{2}}\left(\alpha_{2}+\mu_{2}+c_{2} \beta_{2}+r \mu_{1}\right)$, a possible maximum $p_{2}$ associated with $z_{1}=0$ and $z_{2} \rightarrow \infty$. Subsequently, we construct a sequence of $\operatorname{set}\left(z_{1}^{k}, z_{2}^{k}, p_{1}^{k}, p_{2}^{k}\right), k=1,2,3 \cdots$ in the way of orderly and repeatedly solving

$$
\begin{gathered}
F_{1}\left(z_{1}^{k}\right)=\frac{\left(p_{1}^{k-1}-c_{1}\right)-r\left(p_{2}^{k-1}-c_{2}\right)}{\left(p_{1}^{k-1}+h\right)-r\left(p_{2}^{k-1}-c_{2}\right)} \\
F_{2}\left(z_{2}^{k}\right)=\frac{p_{2}^{k-1}-c_{2}}{p_{2}^{k-1}+h} \\
p_{1}^{k}=\frac{1}{2 \beta_{1}}\left(\alpha_{1}+\mu_{1}+c_{1} \beta_{1}-\Theta_{1}\left(z_{1}^{k}\right)\right) \\
p_{2}^{k}=\frac{1}{2 \beta_{2}}\left(\alpha_{2}+\mu_{2}+c_{2} \beta_{2}-\Theta_{2}\left(z_{2}^{k}\right)+r \Theta_{1}\left(z_{1}^{k}\right)\right)
\end{gathered}
$$

Under this situation, according to the second fact above, monotonous increases of $z_{1}^{k}, p_{1}^{k}$ and monotonous decreases of $z_{2}^{k}, p_{2}^{k}$ are obtained. Plus, because $z_{2}^{k}$ is also bounded below, convergences of the monotonously decreasing $z_{2}^{k}$ is therefore verified and so are the $z_{1}^{k}, p_{1}^{k}$ and $p_{2}^{k}$ as a result of that. In the aftermath, we conclude that the sequence $\left(z_{1}^{k}, z_{2}^{k}, p_{1}^{k}, p_{2}^{k}\right)$ converges to the solution of Eqs.(10)-(13), which is also unique due to the concavity of $E$. Altogether, the following outcome is claimed.

\section{Proposition 1}

(1) $E$ is jointly concave in $z_{1}, z_{2}, p_{1}, p_{2}$ as long as

$$
\left(\left(p_{1}+h\right)-r\left(p_{2}-c_{2}\right)\right) f_{1}\left(z_{1}\right)>1+\frac{1}{2 \beta_{1}}
$$

and

$$
\left(p_{2}+h\right) f_{2}\left(z_{2}\right)>\frac{1}{2 \beta_{2}-1}
$$

(2) Its optimal $z_{1}, z_{2}, p_{1}, p_{2}$ are given in Eqs.(10)-(13) and is unique in

$$
\begin{gathered}
T=\left\{\left(z_{1}, z_{2}, p_{1}, p_{2}\right) \mid 0 \leq z_{1}, z_{2}<\infty,\right. \\
\frac{1}{2 \beta_{1}}\left(\alpha_{1}+c_{1} \beta_{1}\right) \leq p_{1}<\frac{1}{2 \beta_{1}}\left(\alpha_{1}+\mu_{1}+c_{1} \beta_{1}\right), \\
\left.\frac{1}{2 \beta_{2}}\left(\alpha_{2}+c_{2} \beta_{2}\right)<p_{2}<\frac{1}{2 \beta_{2}}\left(\alpha_{2}+\mu_{2}+c_{2} \beta_{2}+r \mu_{1}\right)\right\}
\end{gathered}
$$




\subsection{The Case Considering a Given $z_{1}$}

It is necessary to consider the case of a known $z_{1}$ because the correlation between $z_{1}$ and $E$ is a key factor to examine whether or not our inadequate stock strategy is workable. The $E$ is thus replaced with $E\left(z_{2}, p_{1}, p_{2} \mid z_{1}\right)$ as $z_{1}$ is given, whose Hessian matrix is $J=\left[\begin{array}{ccc}-\left(p_{2}+h\right) f_{2}\left(z_{2}\right) & 0 & 1-F_{2}\left(z_{2}\right) \\ 0 & -2 \beta_{1} & 0 \\ 1-F_{2}\left(z_{2}\right) & 0 & -2 \beta_{2}\end{array}\right]$ with the

following principal minors $\left|J_{11}\right|=-\left(p_{2}+h\right) f_{2}\left(z_{2}\right)<0$ $\left|J_{22}\right|=2 \beta_{1}\left(p_{2}+h\right) f_{2}\left(z_{2}\right)>0$ and $\left|J_{33}\right|=-2 \beta_{1}\left(2 \beta_{2}\left(p_{2}+h\right) f_{2}\left(z_{2}\right)-\left(1-F_{2}\left(z_{2}\right)\right)^{2}\right) \quad<0 \quad$ if $\left(p_{2}+h\right) f_{2}\left(z_{2}\right)>\frac{1}{2 \beta_{2}}$. Thus, with respect to a given $z_{1}$, we draw a conclusion below.

\section{Proposition 2}

The $E\left(z_{2}, p_{1}, p_{2} \mid z_{1}\right)$ is jointly concave in $z_{2}, p_{1}, p_{2}$ as long as $\left(p_{2}+h\right) f_{2}\left(z_{2}\right)>\frac{1}{2 \beta_{2}}$, and its optimal solution is given by Eqs.(11)-(13).

\subsection{The Case Considering a Fixed $p_{1}$ and $p_{2}$}

In practice, giving a fixed $p_{1}$ and $p_{2}$ is quite common in such a competitive market. In this situation, policy makers will face a problem on how to price the two items in advance in order to ensure the existence of optimal $z_{1}, z_{2}$ that maximizes the profit.

The $E$ is thus replaced with $E\left(z_{1}, z_{2} \mid p_{1}, p_{2}\right)$ when $p_{1}$ and $p_{2}$ are fixed, whose Hessian matrix is $G=\left[\begin{array}{cc}\left(r\left(p_{2}-c_{2}\right)-\left(p_{1}+h\right)\right) f_{1}\left(z_{1}\right) & 0 \\ 0 & -\left(p_{2}+h\right) f_{2}\left(z_{2}\right)\end{array}\right]$ with the following principal minors $\left|G_{11}\right|=\left(r\left(p_{2}-c_{2}\right)-\left(p_{1}+h\right)\right) f_{1}\left(z_{1}\right)$

and $\left|G_{22}\right|=-\left(r\left(p_{2}-c_{2}\right)-\left(p_{1}+h\right)\right) f_{1}\left(z_{1}\right)\left(p_{2}+h\right) f_{2}\left(z_{2}\right)$. Clearly, $G$ will be negative-definite if

$$
r\left(p_{2}-c_{2}\right)<\left(p_{1}+h\right) \text {. }
$$

Moreover, its optimal necessary condition Eq.(10) reveals that only $r\left(p_{2}-c_{2}\right) \leq\left(p_{1}-c_{1}\right)$ generates a non-negative $F_{1}\left(z_{1}\right)$, a requirement for a non-negative optimal $z_{1}$. To explain this, we re-regard the term $\left(p_{2}-c_{2}\right) r \Theta_{1}\left(z_{1}\right)$ in Eq.(5) as the amount of $\Theta_{1}\left(z_{1}\right)$ shifting to the second period with a profit margin $r\left(p_{2}-c_{2}\right)$, and this helps realize why only $r\left(p_{2}-c_{2}\right) \leq\left(p_{1}-c_{1}\right)$ yields a non-negative optimal $z_{1}$. On the contrary, $r\left(p_{2}-c_{2}\right)>\left(p_{1}-c_{1}\right)$ implies a higher profit margin in the second period; that way, in pursuit of a higher profit, the error demand in the first period should be utterly discarded, that is, set optimal $z_{1}=0$. Consequently, a basic fundamental on how to price these two items is acquired as follows.

\section{Proposition 3}

(1) $E\left(z_{1}, z_{2} \mid p_{1}, p_{2}\right)$ is jointly concave in $z_{1}, z_{2}$ if the pre-set $p_{1}, p_{2}$ satisfy $r\left(p_{2}-c_{2}\right)<\left(p_{1}+h\right)$.

(2) If the pre-set $p_{1}, p_{2}$ satisfy $r\left(p_{2}-c_{2}\right) \leq\left(p_{1}-c_{1}\right)$, then the optimal $z_{1}, z_{2}$ are obtained according to Eqs.(10)-(11).

(3)If the pre-set $p_{1} \quad, \quad p_{2}$ satisfy $p_{1}-c_{1}<r\left(p_{2}-c_{2}\right)<\left(p_{1}+h\right)$, then the optimal $z_{1}$ is set to be 0 , and the optimal $z_{2}$ is obtained according to Eq.(11).

\section{Examples}

The following examples are respectively responding to the cases of $E, E\left(z_{2}, p_{1}, p_{2} \mid z_{1}\right), E\left(z_{1}, z_{2} \mid p_{1}, p_{2}\right)$ and the impact on $E$ caused by an increasing $\alpha_{2}$, an increasing $\mu_{2}$ and a cheaper $c_{2}$. The values of the parameters are as follows. For item $i, i=1,2, \quad f_{i}\left(\varepsilon_{i}\right)=\lambda_{i} e^{-\lambda_{i} \varepsilon_{i}}$, $\alpha_{i}=300, \beta_{i}=5, c_{i}=10, \lambda_{i}=0.03$ and $h=5$. The first example, illustrated by Table 1, is showing the optimal $p_{1}, p_{2}, E, z_{1}, z_{2}, Q_{1}$ and $Q_{2}$ with respect to the various $r$ from $r=0$ to $r=1$. The second example points out the optimal $p_{1}, p_{2}, E\left(z_{2}, p_{1}, p_{2} \mid z_{1}\right), z_{2}, Q_{1}, Q_{2}$ from $z_{1}=0$ to $z_{1}=90$ at $r=0.7$ in Table 2 . The third example, illustrated by Table 3 , displays the optimal $z_{1}, z_{2}, Q_{1}, Q_{2}$ and $E\left(z_{1}, z_{2} \mid p_{1}, p_{2}\right)$ from $p_{2}=30$ to $p_{2}=45$ at a fixed $p_{1}=35$ as $r=0.7$. And the last example, illustrated by Table 4 at where the effect of an increasing primary demand is made by increasing $\alpha_{2}$ by $10 \%$ at a time, the effect of an increasing error demand is made by increasing $\mu_{2}$ by $10 \%$ 
at a time and the effect of a cheaper purchasing cost is made by reducing $c_{2}$ by $10 \%$ at a time, is examining how the three triggered effects would respectively and simultaneously influence the $E$ as $r=0.7$.

Table 1 first attests that our profit performances always outreach the classical newsvendor setting whose profit is $E=6980.59$ shown by setting $r=0$ in Table 1 . Also, Table 1 discloses a result consistent with our intuition that a bigger $r$ infers a larger shifting demand to the second period, which therefore allows a higher price $p_{2}$ for the second item and consequently yields a better total profit $E$. Thus, in response to a high shifting demand rate, policy makers are urged to reduce the first item's inventory prompting more demand to the second period, and then to increase the second item's inventory satisfying the extra sales. And this explains why Table 1 suggests that $p_{1}, z_{1}$ and $Q_{1}$ decrease, while $p_{2}, z_{2}, Q_{2}$ and $E$ increase as $r$ increases. We note that, for the cases of $r=0.9$ and $r$ $=1$ in Table 1 , the results indicate that there is no optimal interior point $\left(z_{1}, z_{2}, p_{1}, p_{2}\right)$ in $T$ respect to the two cases, implying that optimal values of the two cases will respectively occur at the boundary of $T$. And this makes the two cases the problem of $E\left(z_{2}, p_{1}, p_{2} \mid z_{1}\right)$ at $z_{1}=0$. Then, the obtained optimal values of $p_{1}$ and $p_{2}$ of the two cases are supposed to be in line with the $p_{1}-c_{1}<r\left(p_{2}-c_{2}\right)$ as mentioned in Proposition 3. Plus, compared to $p_{1}$, Table 1 shows that there always is a higher $p_{2}$ whatsoever.

Table 1. Optimal values with respect $r$

\begin{tabular}{ccccccccc}
\hline$r$ & $p_{1}$ & $p_{2}$ & $E$ & $z_{1}$ & $z_{2}$ & $Q_{1}$ & $Q_{2}$ \\
\cline { 2 - 7 } 0.0 & 37.15 & 37.15 & 6980.59 & 34.44 & 34.44 & 148.70 & 148.70 \\
0.1 & 37.06 & 37.28 & 7014.00 & 32.14 & 34.54 & 146.82 & 149.42 \\
0.2 & 36.96 & 37.43 & 7050.09 & 29.62 & 34.66 & 144.81 & 150.26 \\
0.3 & 36.84 & 37.61 & 7089.39 & 26.84 & 34.80 & 142.63 & 151.23 \\
0.4 & 36.70 & 37.82 & 7132.63 & 23.73 & 34.97 & 140.24 & 152.41 \\
0.5 & 36.51 & 38.08 & 7180.81 & 20.17 & 35.17 & 137.60 & 153.86 \\
0.6 & 36.27 & 38.42 & 7235.50 & 15.97 & 35.43 & 134.62 & 155.72 \\
0.7 & 35.92 & 38.88 & 7299.32 & 10.73 & 35.78 & 131.15 & 158.27 \\
0.8 & 35.33 & 39.62 & 7377.63 & 3.45 & 36.34 & 126.81 & 162.30 \\
0.9 & 35.00 & 40.23 & 7475.95 & 0.00 & 36.79 & 125.00 & 165.65 \\
1.0 & 35.00 & 40.57 & 7577.28 & 0.00 & 37.04 & 125.00 & 167.53 \\
\hline
\end{tabular}

Table 2. Optimal values with respect to $z_{1}$ as $r=0.7$

\begin{tabular}{cccccc}
\hline$z_{1}$ & $p_{1}$ & $p_{2}$ & $E\left(z_{2}, p_{1} p_{2} \mid z_{1}\right)$ & $z_{2}$ & $Q_{1}$ \\
0 & 35.00 & 39.54 & 7276.71 & 36.28 & 125.00 \\
10 & 35.86 & 38.92 & 7299.22 & 35.81 & 130.68 \\
20 & 36.50 & 38.46 & 7284.25 & 35.46 & 137.48 \\
30 & 36.98 & 38.12 & 7238.26 & 35.20 & 145.11 \\
40 & 37.33 & 37.87 & 7167.49 & 35.00 & 153.35 \\
50 & 37.59 & 37.68 & 7077.38 & 34.86 & 154.48 \\
60 & 37.78 & 37.54 & 6972.41 & 34.75 & 152.05 \\
70 & 37.93 & 37.44 & 6856.12 & 34.67 & 151.09 \\
80 & 38.03 & 37.36 & 6731.30 & 34.61 & 150.37 \\
90 & 38.11 & 37.31 & 6600.06 & 34.56 & 159.85 \\
\hline
\end{tabular}


Table 3. Optimal values with respect to $p_{2}$ at $p_{1}=35$ as $r=0.7$

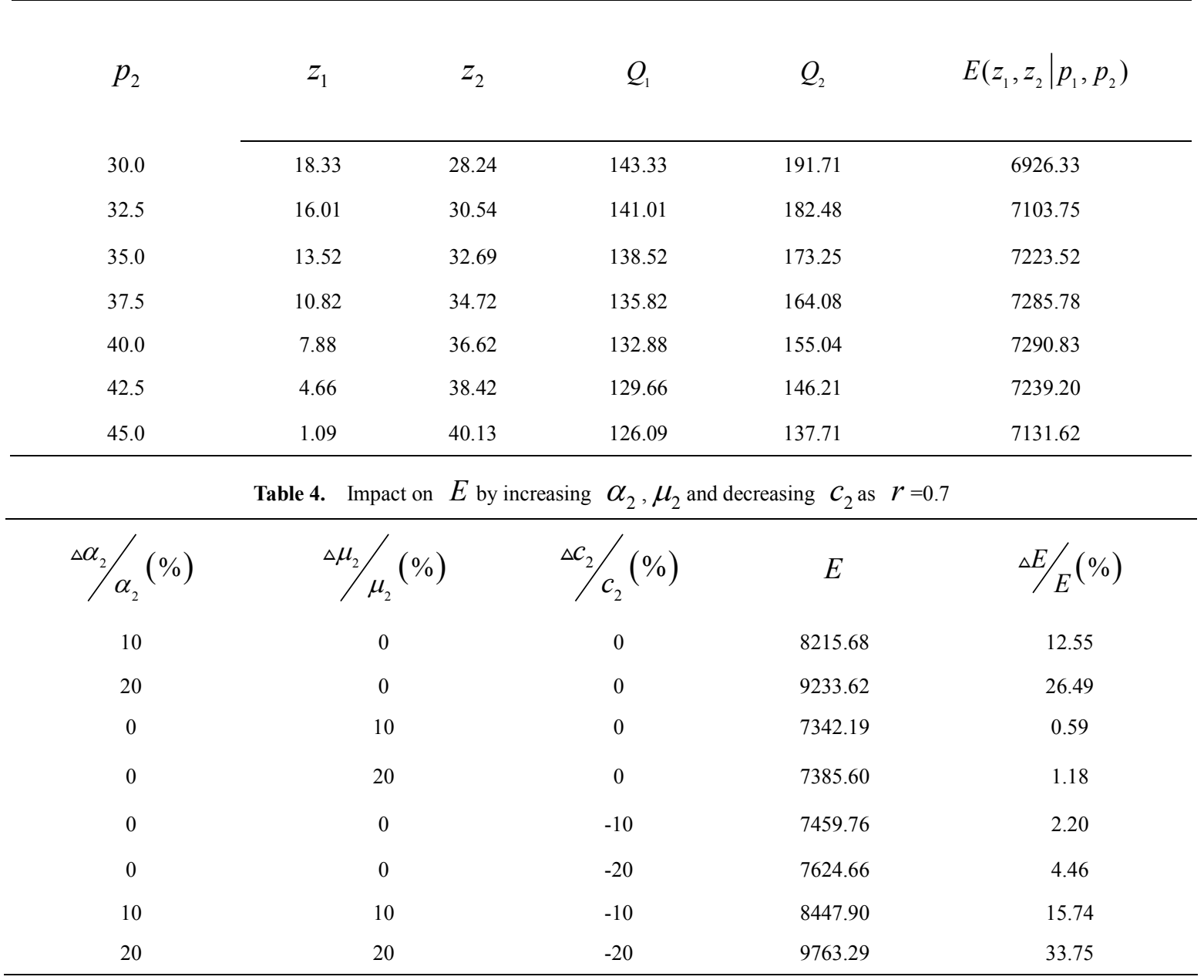

Through the correlation between $E\left(z_{2}, p_{1}, p_{2} \mid z_{1}\right)$ and the various values of $z_{1}$, the purpose of Table 2 is to convince that our inadequate stock strategy is indeed workable since the Table 2 shows that $E\left(z_{2}, p_{1,} p_{2} \mid z_{1}\right)$ first increases from 7276.71 at $z_{1}=0$ to a climax 7299.72 at $z_{1}=10$ and finally to a bottom 6600.06 at $z_{1}=90$. This obviously implies that our first item's under-stock strategy really is practicable and realistic because the profit peaks at a small value $z_{1}=10$. Further, it is shown that just 70 percent of the first item's unsatisfied demand shifting to the second period enables a better total profit $E$ in comparison with that in the classical newsvendor setting.

Table 3 pinpoints how our system will react to a fixed $p_{1}=35$ and a variety values of $p_{2}$ from $p_{2}=30$ to $p_{2}$ $=45$ as $r=0.7$, showing that $E\left(z_{1}, z_{2} \mid p_{1}, p_{2}\right)$ increases at first and then decreases after $p_{2}=40$. This result is consistent with that in Table 1 and is conceivable because that $p_{2} \geq p_{1}$ implies a higher profit margin in the second period. However, an overpriced $p_{2}$, taking $p_{2}=42.5$ or $p_{2}=45.0$ in Table 3 for example, will undermine $E\left(z_{1}, z_{2} \mid p_{1}, p_{2}\right)$ because it decreases the amount of the second item's deterministic demand, resulting in a less optimal inventory $Q_{2}$ and thereby a worse $E\left(z_{1}, z_{2} \mid p_{1}, p_{2}\right)$.

From Table 4, we learn that a $20 \%$ increase in $\alpha_{2}$ can engender a massive increase in $E$ by $26.49 \%$, but a $20 \%$ increase in $\mu_{2}$ can only ignite a $1.18 \%$ increase in $E$. Also, $E$ is slightly increased by $4.46 \%$ after a $20 \%$ reduction in $c_{2}$. The first outcome of Table 4 therefore highlights the importance of connecting product extension with our two-period newsvendor setting since the higher primary demand $\alpha_{2}$ is proven to contribute most profit increment to $E$. Not surprisingly, the three-combined effect yields a dramatic increase in $E$ by $33.75 \%$, and clearly its generated profit $E=9763.29$ makes our strategic setting a great significance in comparison with the classical setting 
whose profit is only $E=6980.59$ as shown in Table 1 .

\section{Conclusion}

This study investigated a two-period newsvendor setting with product extension and shifting demand concerns, during which the item in the second period is a product extension of the item in the first period. An inadequate inventory of the first item is purposely placed such that parts of its unsatisfied demand will shift to the second period; and hopefully, it would induce more sales towards the second item.

Contributions and managerial insights obtained from the study are summarized as follows: (1) even though none of the three could-be-triggered effects occurs, the examples conducted still confirmed our strategic setting's remarkable profit performances by comparison with the classical newsvendor setting; (2) in response to an item with high shifting demand rate, the experiments unveiled that the error demand of the first item sometimes should be totally sacrificed; (3) we provided a basic fundamental to set the two items' selling prices that maximize the total profit; (4) it was shown that a pricy second item, compared to the first item, is indispensable to our strategic setting since it help reach a better total profit; (5)an increasing primary demand, an increasing error demand and a cheaper purchasing cost in the second period were most anticipated in the course of the system; (6)more importantly, with aids of the examples conducted, our two-period newsvendor setting, incorporating with the product extension and the strategy of an inadequate stock in the first selling period, was proven realistic and practicable.

For future researches, it is useful to extend our two-period setting to a multi-period one that allows demand shifting between any two consecutive periods because most newsvendor-type products possess the property of homogeneity and consistency. Meanwhile, since a supply chain setting is regarded as a more realistic and practical system in such a competitive market, it is feasible to modify our system to a one-manufacturer-one-retailer or a one-manufacturer-multiple-retailer type of supply chain. Furthermore, a short duration between any two consecutive periods might be unable to fulfill our expectations of enticing more sales in the second period; a long duration, however, could probably impair the functions of the shortage-making strategy since it will deplete customers' waiting patience. Therefore, how to determine an appropriate duration between any two consecutive periods is also a worthwhile topic.

\section{REFERENCES}

[1] F. J. Arcelus, S. Kumar, G. Srinivasan.Evaluating manufacturer's buyback policies in a single-period two-echelon framework underprice-dependent stochastic demand, Omega, Vol.36, 808-824,2008.

[2] M. Zhang, P. C. Bell, G. Cai, X. Chen.Optimal fences and joint price and inventory decisions in distinct markets with demand leakage, European Journal of Operational Research, Vol.204, 589-596, 2010.

[3] Z. Yao, S. C. H. Leung, K. K. Lai. Analysis of the impact of price-sensitivity factors on the returns policy in coordinating supply chain, European Journal of Operational Research, Vol.187, 275-282, 2008.

[4] E. J. Jr. Lodree, Y. Kim, W. Jang. Time and quantity dependent waiting cost in a newsvendor problem with backlogged shortages, Mathematical and Computer Modelling,Vol.47, 60-71, 2008.

[5] S. G. Johansen, A. Thorstenson. An inventory model with Poisson demands and emergency orders, International Journal of Production Economics,Vol.56-57: 275-289, 1998.

A. Halati, Y. He. Analysis of supply chains with quantity based fixed incentives, European Journal of Operational Research,Vol.202, 214-222, 2010.

[6] H. S. Lau, A. H. L. Lau. Manufacturer's pricing strategy and return policy for a single-period commodity, European Journal of Operational Research,Vol.116, 291-304, 1999.

[7] C. S. Chung, J. Flynn. A newsboy problem with reactive production, Computer \& Operations Research,Vol.28, 751-765, 2001.

[8] B. Das, M. Maiti. An application of bi-level newsboy problem in two substitutable items under capital cost, Applied Mathematics and Computation,Vol.190, 410-422, 2007.

[9] Bose, P. Anand. On returns policies with exogenous price, European Journal of Operational Research,Vol.178, 782-788, 2007.

[10] Y. Serin. Competitive newsvendor problems with the same Nash and Stackelberg solutions, Operations Research Letters,Vol.35, 83-94, 2007.

[11] C. Shi, B. Chen.Pareto-optimal contracts for a supply chain with satisficing objectives, Journal of the Operational Research Society,Vol.58, 751-759, 2007.

[12] S. Yang, C. V. Shi, X. Zhao. Optimal ordering and pricing decisions for a target oriented newsvendor, Omega,Vol.39, 110-115, 2011

[13] H. Wang, C. T. Tung. Construction of a model towards EOQ and pricing strategy for gradually obsolescent products, Applied Mathematics and Computation,Vol.217, 6926-6933, 2011.

[14] P. S. You, T. C. Chen. Dynamic pricing of seasonal goods with spot and forward

[15] purchase demands, Computers and Mathematics with Applications, Vol.54, 490-498, 2007.

[16] G. T. Thowsen. A dynamic, non-stationary inventory problem for a price/quantity setting firm, Naval Research Logistics Quarterly,Vol.22, No. 3, 461-476,1975.

[17] N. C. Petruzzi, M. Dada. Pricing and the newsvendor problem: A review with extensions, Operations Research,Vol. 47, No. 2, 183-194, 1999. 